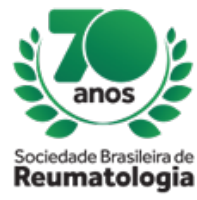

\title{
DEPRESSION AND PSYCHOSIS IN SYSTEMIC LUPUS ERYTHEMATOSUS: A CASE REPORT
}

Laura Alicia Morais Lima Oliveira (Universidade Federal do Rio Grande do Norte, CAICÓ, RN, Brasil), Ana Olivia Dantas (Universidade Federal do Rio Grande do Norte, Caicó, RN, Brasil), Lauanda Ênia Medeiros Rocha (Universidade Federal do Rio Grande do Norte, Caicó, RN, Brasil), Emilie Queiroga Queiroga (Universidade Federal do Rio Grande do Norte, Caicó, RN, Brasil), Heron Alves Vale (Universidade Federal do Rio Grande do Norte, Caicó, RN, Brasil), Pedro Augusto Dias Timoteo (Universidade Federal do Rio Grande do Norte, Caicó, RN, Brasil)

\section{BACKGROUND}

Systemic lupus erythematosus (SLE) is a chronic inflammatory disease of autoimmune origin, that can affect several systems of the human organism. The neuropsychiatric manifestations of SLE are less frequent in the diagnosed population. However, they can cause important alterations, such as disorders of the motricity, mood and behavior.

\section{CASE REPORT}

A 38-year-old female patient was diagnosed with SLE in 2018, when she started a clinical picture with skin lesions in photoexposed areas, arthralgia in elbows, knees and ankles, and malar rash. Laboratory evaluation showed serum ANA positive (1:640 - nuclear fine speckled pattern), positive anti-Ro (>240 $\mathrm{U} / \mathrm{mL}$ ) and anti-La $(19 \mathrm{U} / \mathrm{mL})$. Initial therapy consisted of hydroxychloroquine and prednisone. During follow-up, azathioprine was also incorporated into the scheme. Pacient evolved with attenuation of arthralgias and reduction of cutaneous lesions. She reported on one of the appointments hypothymia, anhedonia and difficulty concentrating on daily tasks, in addition to impaired sleep and easy crying during the day. Referred to psychiatry, the patient also claimed to have visual hallucinations, consisting of an unknown child and man, besides hearing voices that encouraged her to attempt suicide and wander aimlessly through the city. Hallucinations were more frequent when she felt agitated or irritated. She still reported a violent relationship with her husband and mother, having already planned suicide a few times. At the time of the consultation, she had no immediate plans to take her own life and claimed to have expectations for the future, especially in regard to her children, her main protective factor. With no previous history of psychotic symptoms or manic expression, and the hypothesis of hypothyroidism excluded by laboratory tests, the possibility of the symptoms being consequences from SLE was strengthened. Sertraline and risperidone were prescribed, which, around one month, led to improvement of the patient's mood and decreased frequency of hallucinations. Risperidone was replaced by haloperidol in an attempt to completely stop the patient's hallucinations. She continues to be evaluated by rheumatology and psychiatry, to follow the treatment and evolution of the symptoms.

\section{CONCLUSION}

The neuropsychiatric manifestations of SLE are reported in the literature, but infrequently when compared to other signs and symptoms. Depression is more easily identified, as opposed to psychosis, an uncommon phenomenon. These clinical entities presuppose a management with other medical specialties and professional categories so that the patient is benefited and presents significant improvement in the course of his disease. 